\title{
Impact of the Maze operation on the progression of mild functional tricuspid regurgitation
}

\author{
Hyung Gon Je, MD, ${ }^{\mathrm{a}}$ Hyun Song, MD, ${ }^{\mathrm{a}}$ Sung Ho Jung, MD, ${ }^{\mathrm{a}}$ Suk Jung Choo, MD, ${ }^{\mathrm{a}}$ Jong Min Song, MD, ${ }^{\mathrm{b}}$ Duk Hyun Kang, MD, \\ Sung Cheol Yun, PhD, ${ }^{\mathrm{c}}$ Cheol Hyun Chung, MD, ${ }^{\mathrm{a}}$ Jae Kawn Song, MD, ${ }^{\mathrm{b}}$ and Jae Won Lee, MD ${ }^{\mathrm{a}}$
}

\begin{abstract}
Objectives: In patients having mitral valve surgery, concomitant surgery for mild functional tricuspid regurgitation remains the subject of debate. This study examined the effect of Maze operation and tricuspid valve repair on postoperative functional tricuspid regurgitation progression.

Methods: The study retrospectively analyzed 250 patients ( 86 men, 164 women) with mild functional tricuspid regurgitation (grade 2) who had mitral valve surgery between January 1994 and July 2006. Based on follow-up data, patients were defined as either stable $(n=209,83.6 \%)$ or aggravated $(n=41,16.4 \%)$. Predictors for significant tricuspid regurgitation development were identified using Cox regression analysis.
\end{abstract}

Results: The mean follow-up time was $62.6 \pm 39.8$ months after surgery. Although most mitral valve procedures were successful, there was an increase in the incidence of significant functional tricuspid regurgitation overall from immediately postoperative to final assessment $(5.2 \%$ to $16.4 \%, P<0.01)$. Univariate analysis showed that old age, shorter aortic crossclamping time, and omission of Maze operation were associated with functional tricuspid regurgitation progression. Multivariate analysis showed that older age (adjusted hazard ratio, 1.05; 95\% confidence interval, 1.02 to 1.08), a rheumatic etiology of the mitral valve disease (adjusted hazard ratio, 2.31; 95\% confidence interval, 1.21 to 4.42), and no Maze operation (adjusted hazard ratio, 7.90; 95\% confidence interval, 1.90 to 32.86) were independent predictors of mild functional tricuspid regurgitation progression. For the 168 patients with preoperative atrial fibrillation, Maze operation improved the tricuspid regurgitation-free survival significantly $(P<.01)$ but tricuspid valve repair showed no significant difference.

Conclusions: Mild functional tricuspid regurgitation can progress postoperatively despite successful mitral valve surgery. Although tricuspid valve repairs alleviate progression of functional tricuspid regurgitation, concomitant Maze operation is a more powerful protective factor against mild functional tricuspid regurgitation progression.

Mitral valvular disease often causes functional tricuspid regurgitation (FTR), which is associated with right ventricular failure and pulmonary hypertension. Some reports suggest that tricuspid regurgitation (TR) can be improved after successful mitral valve (MV) surgery ${ }^{1}$ or percutaneous mitral valvuloplasty. ${ }^{2}$ However, other reports recommend that significant FTR should be repaired due to increased postoperative morbidity and mortality after left-sided valvular surgery. ${ }^{3,4}$

The fate, risk factors, and surgical indications for FTR after mitral and/or aortic valve surgery have been reported..$^{5-8}$ Moderate to severe FTR is generally corrected during MV surgery as significant FTR can increase mortality and morbidity. However, for the mild FTR, the decision to perform surgery remains debatable in terms of when and how to treat the tricuspid valve (TV). Although it is not uncommon to

\footnotetext{
From the Departments of Cardiovascular Surgery, ${ }^{\mathrm{a}}$ Cardiology, ${ }^{\mathrm{b}}$ and Preventive Medicine, ${ }^{\mathrm{c}}$ Asan Medical Center, College of Medicine, University of Ulsan, Seoul, Korea.

Received for publication Dec 13, 2007; revisions received July 13, 2008; accepted for publication Aug 5, 2008.

Address for reprints: Hyun Song, MD, Department of Cardiovascular Surgery, Asan Medical Center, University of Ulsan, College of Medicine, 388-1 Poongnap2dong, Songpa-gu, Seoul 138-736, South Korea (E-mail: cs3580@amc.seoul.kr).

J Thorac Cardiovasc Surg 2008;136:1187-92

$0022-5223 / \$ 34.00$

Copyright (C) 2008 by The American Association for Thoracic Surgery

doi:10.1016/j.jtcvs.2008.08.009
}

encounter a patient with MV disease and mild FTR, relatively few clinical studies provide information that might assist in managing this situation. To our knowledge, no studies have investigated surgical outcomes in patients with mild FTR exclusively.

Several surgical options for FTR-associated MV disease have been reported, including MV surgery, ${ }^{1}$ which expects to improve FTR as the result of its performance, aggressive TV repair to prevent TR progression even when the initial TR was mild, ${ }^{6}$ and MV surgery with a Maze operation (MAZE) to prevent progression of functional TR. ${ }^{9} \mathrm{We}$ hypothesize that concomitant MAZE and TV repair can prevent progression of mild FTR in patients having MV surgery.

The present study analyzed surgical outcomes in patients with mild FTR having MV surgery. The study examined the effect of MAZE and TV repair on postoperative TR progression. The study also sought to identify factors associated with TR progression that may act as indicators for concomitant surgical treatment of patients with mild FTR having MV surgery.

\section{MATERIALS AND METHODS \\ Study Population}

From January 1994 to July 2006, 2417 patients had MV surgery at the Asan Medical Center, Seoul, South Korea. Of those, excluded from 


$$
\begin{aligned}
& \text { Abbreviations and Acronyms } \\
& \text { ACC }=\text { aortic crossclamping } \\
& \mathrm{AF}=\text { atrial fibrillation } \\
& \text { FTR = functional tricuspid regurgitation } \\
& \text { MAZE }=\text { Maze operation } \\
& \text { MR }=\text { mitral regurgitation } \\
& \text { MV = mitral valve } \\
& \mathrm{TAP}=\text { tricuspid annuloplasty } \\
& \text { TR = tricuspid regurgitation } \\
& \mathrm{TV}=\text { tricuspid valve }
\end{aligned}
$$

consideration for this study were 1037 patients who had combined surgery (such as coronary bypass, aortic valve surgery, or thoracic aorta surgery) and patients who were followed for less than 12 months. Of the remaining 1380 patients who had only MV surgery and were followed for 12 months or more at Asan Medical Center, 250 consecutive patients showing mild FTR according to preoperative echocardiography were recruited for the study. Data regarding surgery were prospectively collected, and a thorough echocardiographic follow-up was performed by a cardiologist from our institute. This study was approved by our institution's ethical committee/institutional review board. The informed consent was waived by our institution's ethical committee/institutional review board owing to the retrospective nature of our study.

\section{Echocardiographic Evaluation}

Transthoracic echocardiographic evaluation was performed before and after surgery, and then annually during follow-up. Two-dimensional echocardiography and Doppler color flow imaging were performed on all patients using a Hewlett-Packard Sonos 2500 or 5500 imaging system equipped with a $2.5-\mathrm{MHz}$ transducer (Hewlett-Packard, Andover, Mass). Preoperative transesophageal echocardiography was routinely performed to evaluate MV morphology.

TR was evaluated using the apical 4-chamber view. TR was graded as trace, mild, moderate, or severe when the jet area occupied $<10 \%, 10 \%$ to $20 \%, 20 \%$ to $33 \%$, or $>33 \%$ of the right atrial area, respectively. During the follow-up, development of moderate to severe TR was defined as significant TR and was also termed " aggravated FTR." Postoperative improvement or maintenance of preoperative mild FTR was defined as "stable FTR." Based on these definitions, there were 209 (83.6\%) patients with stable FTR and $41(16.4 \%)$ patients with aggravated FTR. Right ventricular systolic pressures were estimated using continuous-wave Doppler data and the simplified Bernoulli equation $\left(4 \times\right.$ [peak TR velocity] $\left.{ }^{2}\right)$ with 10 $\mathrm{mm} \mathrm{Hg}$ added for the estimated right atrial pressure. ${ }^{10}$ Residual mitral regurgitation (MR) was defined as more than mild MR at follow-up echocardiography, and residual MS was defined as less than $1.5 \mathrm{~cm}^{2}$ of the MV area during follow-up.

\section{Surgical Procedures}

All patients had MV surgery without aortic valve or coronary surgery. MV replacement was performed in 147 patients (58.8\%) and MV repair in $103(41.2 \%)$ patients. MV replacement involved bileaflet mechanical prostheses $(n=127,86.4 \%)$ or bioprostheses $(n=20,13.6 \%)$. MV repair was attempted whenever MV pathology was favorable, and MV replacement was performed only when the MV was severely destructed or MV repair was unsatisfactory on intraoperative transesophageal echocardiography. A median sternotomy approach was used for most patients $(225,90 \%)$ and a right anterolateral minithoracotomy approach using the AESOP 3000 system (Computer Motion, Inc., Santa Barbara, CA) was used in the most recent $25(10 \%)$ patients. The recent minimally invasive approach has several advantages such as stable thoracoscopic view, easily controlled with surgeon's voice, and reduction of one surgical assistant. Conventional ascending aorta and bicaval cannulation was used for the sternotomy approach, and the minimally invasive approach involved peripheral cannulation via the right internal jugular vein, right femoral vein, and right femoral artery.

TV repair was performed in $82(32.8 \%)$ patients. Of those, 55 (22.0\%) had suture annuloplasty and $27(10.8 \%)$ had ring annuloplasty. The decision to perform a TV repair and the choice of repair technique was at the attending surgeon's discretion; ring annuloplasty was generally used for more severe tricuspid annular dilatation. Ring annuloplasty involved a Carpentier ring (Edwards Lifesciences, Irvine, Calif) in 4 patients and a Duran ring (Medtronic, Minneapolis, Minn) in 23 patients, and the most common ring size was $27 \mathrm{~mm}$. Kay annuloplasty (bicuspidalization) or a De Vega procedure (from the anteroseptal to the posteroseptal commissure) was performed for suture annuloplasty according to surgeon preference.

Concomitant procedures consisted of MAZE in 73 (29.2\%) patients, atrial septal defect repair in 7 patients, and left atrial myxoma resection in 2 patients. Five patients had a history of percutaneous mitral valvuloplasty. Among 73 MAZE procedures, between 1997 and 1998, 2 patients had a classic cut-and-sew Cox-Maze III, and since 1998, 71 patients had simplified MAZE, which was modified by the authors. ${ }^{11}$

As MAZE has been performed since 1997 and its application was totally at the surgeon's discretion, only a small number of patients had the MAZE in this study. Recently, the MAZE was rather generally performed to treat atrial fibrillation (AF) in our institution; however, some authors in this study have been prudent to perform a MAZE especially in conjunction with MV replacement using a mechanical prosthesis.

Thirty-eight (15.2\%) patients had previous cardiac surgery.

\section{Clinical Follow-up}

Clinical follow-up ranged from 12 to 160 months. Data were obtained during visits to the outpatient clinic or by telephone interview. Patient clinical status and echocardiographic results were obtained from cardiologists at our institute. Patients had an echocardiography assessment during the week after surgery, at postoperative 6 months, and then annually during followup. Because patients who had echocardiographic follow-up at local clinics were excluded from the study design, a complete echocardiogram follow-up could be achieved in these study populations. Normal sinus rhythm restoration after MAZE was defined as a presence of transmitral A-wave at the last echocardiographic follow-up.

\section{Statistical Analysis}

Continuous variables are presented as mean \pm standard deviation, and categorical variables as percentages or numbers. For univariate analyses, preoperative and operative variables were analyzed using the KaplanMeier method or the Cox regression model to investigate the influence of these variables on FTR progression during follow-up. The difference of significant TR development at the immediate postoperative period, at postoperative 1 year, and the most recent follow-up was assessed using McNemar's test. Independent predictors were determined using Cox's multivariable analysis involving a backward elimination procedure. The proportional hazards assumption was confirmed by examination of $\log$ $\left(-\log\right.$ [survival]) curves and by testing partial (Schoenfeld) residuals, ${ }^{12}$ and no relevant violations were found. All $P$ values were 2 -sided. Statistical analysis was performed using SPSS version 12.0 for Windows (SPSS Inc, Chicago, Ill).

\section{Declaration}

All authors had full access to the data and take responsibility for its integrity. All authors have read the manuscript and accept the contents. 
TABLE 1. Preoperative and operative characteristics for patients overall and for patients with stable and aggravated tricuspid regurgitation

\begin{tabular}{|c|c|c|c|c|}
\hline & $\begin{array}{c}\text { Overall } \\
(\mathbf{n}=\mathbf{2 5 0})\end{array}$ & $\begin{array}{c}\text { Stable } \\
(\mathrm{n}=\mathbf{2 0 9})\end{array}$ & $\begin{array}{c}\text { Aggravated } \\
(\mathrm{n}=41)\end{array}$ & $\begin{array}{c}P \\
\text { value }\end{array}$ \\
\hline \multicolumn{5}{|l|}{ Preoperative } \\
\hline Age (y) & $50.0 \pm 13.1$ & $48.9 \pm 13.2$ & $55.8 \pm 10.6$ & $<.01^{*}$ \\
\hline Female & $65.6 \%(164)$ & $60.8 \%(127)$ & $90.2 \%(37)$ & $<.01 \dagger$ \\
\hline Atrial fibrillation & $67.2 \%(168)$ & $64.6 \%(135)$ & $80.5 \%(33)$ & $.13 \dagger$ \\
\hline Rheumatic etiology & $49.6 \%(124)$ & $47.8 \%(100)$ & $58.5 \%(24)$ & $.12 *$ \\
\hline $\mathrm{EF}(\%)$ & $58.1 \pm 9.4$ & $58.0 \pm 9.6$ & $58.3 \pm 8.3$ & $>0.1^{*}$ \\
\hline LA size $(\mathrm{mm})$ & $59.4 \pm 10.9$ & $59.2 \pm 11.2$ & $60.6 \pm 9.0$ & $>0.1^{*}$ \\
\hline sPAP (mm Hg) & $49.2 \pm 17.8$ & $49.5 \pm 17.4$ & $47.7 \pm 20.0$ & $>0.1^{*}$ \\
\hline MV pathology & & & & $.12 \dagger$ \\
\hline Mitral stenosis & $44.0 \%(110)$ & $41.1 \%(86)$ & $58.5 \%(24)$ & \\
\hline $\begin{array}{l}\text { Mitral } \\
\quad \text { regurgitation }\end{array}$ & $56.0 \%(140)$ & $58.9 \%(123)$ & $41.5 \%(17)$ & \\
\hline \multicolumn{5}{|l|}{ Operative } \\
\hline CPB time (min) & $128.9 \pm 47.7$ & $7130.9 \pm 48.3$ & $118.3 \pm 43.6$ & $.14 *$ \\
\hline ACC time (min) & $84.0 \pm 35.4$ & $86.6 \pm 26.6$ & $70.9 \pm 26.6$ & $.02 *$ \\
\hline MV procedure & & & & $0.07 \dagger$ \\
\hline MV repair & $41.2 \%(103)$ & $44.0 \%(92)$ & $26.8 \%(11)$ & \\
\hline MV replacement & $58.8 \%(147)$ & $56.0 \%(117)$ & $73.2 \%(30)$ & \\
\hline Maze procedure & $29.2 \%(73)$ & $34.0 \%(71)$ & $4.9 \%(2)$ & $.01 \dagger$ \\
\hline TV repair & $32.8 \%(82)$ & $35.4 \%(74)$ & $19.5 \%(8)$ & $.07 \dagger$ \\
\hline $\begin{array}{l}\text { Suture } \\
\text { annuloplasty }\end{array}$ & $22 \%(55)$ & $23.4 \%(49)$ & $14.6 \%(6)$ & $\mathrm{NS} \dagger$ \\
\hline $\begin{array}{l}\text { Ring } \\
\quad \text { annuloplasty }\end{array}$ & $10.8 \%(27)$ & $12.0 \%(25)$ & $4.9 \%(2)$ & $\mathrm{NS} \dagger$ \\
\hline
\end{tabular}

$n$, Number; $C P B$, cardiopulmonary bypass; $A C C$, aortic crossclamp; $E F$, ejection fraction; $L A$, left atrium; $s P A P$, systolic pulmonary artery pressure; $M V$, mitral valve; $T V$, tricuspid valve; $N S$, not significant. ${ }^{*}$ Cox regression test. $\dagger$ Log-rank test and KaplanMeier analysis. ‡Mann-Whitney test.

\section{RESULTS}

\section{Perioperative Characteristics}

The patient baseline preoperative and operative characteristics are summarized in Table 1 . There were no significant differences between the stable and aggravated groups in terms of left ventricular ejection fraction, left atrial size, estimated systolic pulmonary artery pressure, cardiopulmonary bypass time, incidence of AF, rheumatic etiology of MV, or incidence of mitral stenosis. Patients in the aggravated group were older, more likely to be female, had longer aortic crossclamping (ACC) time, and were less likely to have had a MAZE than the stable group. The longer ACC time in the stable group reflects that the patients were more likely to have had MV repair, MAZE, and TV repair (Table 1).

\section{Operative Mortality and Morbidities}

The operative mortality of all consecutive patients who had MV surgery including concomitant surgery during study period was $2.1 \%(50 / 2417)$.

Among the 250 patients who enrolled in this study, 9 patients $(3.6 \%)$ had reoperation for bleeding, 5 patients
TABLE 2. Long-term echocardiographic and clinical outcomes

\begin{tabular}{lcccr}
\hline & $\begin{array}{c}\text { Overall } \\
(\mathbf{n = 2 5 0 )}\end{array}$ & $\begin{array}{c}\text { Stable } \\
(\mathbf{n}=\mathbf{2 0 9})\end{array}$ & $\begin{array}{c}\text { Aggravated } \\
(\mathbf{n = 4 1 )}\end{array}$ & $\begin{array}{c}\boldsymbol{P} \\
\text { value }\end{array}$ \\
\hline Residual MR & $5.2 \%(13)$ & $4.8 \%(10)$ & $7.3 \%(3)$ & $>0.1$ \\
Residual MS & $2.4 \%(6)$ & $1.9 \%(4)$ & $4.9 \%(2)$ & $>0.1$ \\
Last EF (\%) & $57.6 \pm 8.1$ & $58.1 \pm 7.5$ & $55.1 \pm 10.1$ & .03 \\
Last LA size (mm) & $49.7 \pm 9.3$ & $48.6 \pm 9.1$ & $55.2 \pm 8.4$ & $<.01$ \\
Last sPAP (mm Hg) & $36.2 \pm 7.5$ & $35.0 \pm 6.3$ & $41.5 \pm 9.8$ & $<.01$ \\
Last NSR & $61.2 \%(153)$ & $70.8 \%(148)$ & $12.2 \%(5)$ & $<.01$ \\
Last Af, Afl, PPM & $38.8 \%(97)$ & $29.2 \%(61)$ & $87.8 \%(36)$ & $<.01$ \\
\hline
\end{tabular}

$n$, Number; $M R$, mitral regurgitation; $M S$, mitral stenosis; $E F$, ejection fraction; $L A$, lef atrium; $S P A P$, systolic pulmonary artery pressure; $N S R$, normal sinus rhythm; $A f$, atrial fibrillation; $A f l$, atrial flutter; $P P M$, permanent pacemaker; $N S$, not significant.

$(2.0 \%)$ had minor sternal wound problems, and 3 patients $(1.2 \%)$ needed pericardial drainage for severe pericardial effusion. A permanent pacemaker was inserted in 2.7\% (2/73) of patients who showed sick sinus syndrome after the MAZE.

\section{Reoperations and Long-Term Clinical Outcomes}

The mean follow-up time was $62.6 \pm 39.8$ months (range 12.0 to 160.3 ). During the follow-up period, only 3 reoperations were required, and these involved correction for severely aggravated TR. The incidences of residual mitral regurgitation and residual mitral stenosis were similar between the stable and aggravated groups (Table 2). Although 9 of the 13 patients with residual MR had moderate MR and 4 of them had severe MR, all patients responded well to medical treatment. Two residual MR cases and 3 residual MS cases were the result of structural deterioration of a bioprosthesis. Only 1 reoperation for an MV was required, and this was in a patient who had mitral annuloplasty with a Duran ring. That patient had a reoperation for severe MS with severe TR at 82 months after the initial operation. Compared with the stable group, the aggravated group had a lower left ventricular ejection fraction, bigger left atrium size, higher systolic pulmonary artery pressure, and greater prevalence of atrial fibrillation on the most recent echocardiographic examination (Table 2).

Although only 5.2\% (13/250) of patients showed significant TR according to immediate postoperative echocardiography, $16.4 \%(41 / 250)$ of patients showed significant TR at the final follow-up (Figure 1), which was a significantly greater figure.

Although MAZE had a significant effect on freedom from significant TR development during follow-up, the effect of tricuspid annuloplasty (TAP) showed marginal significance (Figure 2).

As an application of MAZE and TV repair was totally at the surgeon's discretion, the possibility of preoperative selection bias was concerned. However, the preoperative variables such as age, left ventricular ejection fraction, and systolic pulmonary pressure were not significantly different between groups. 


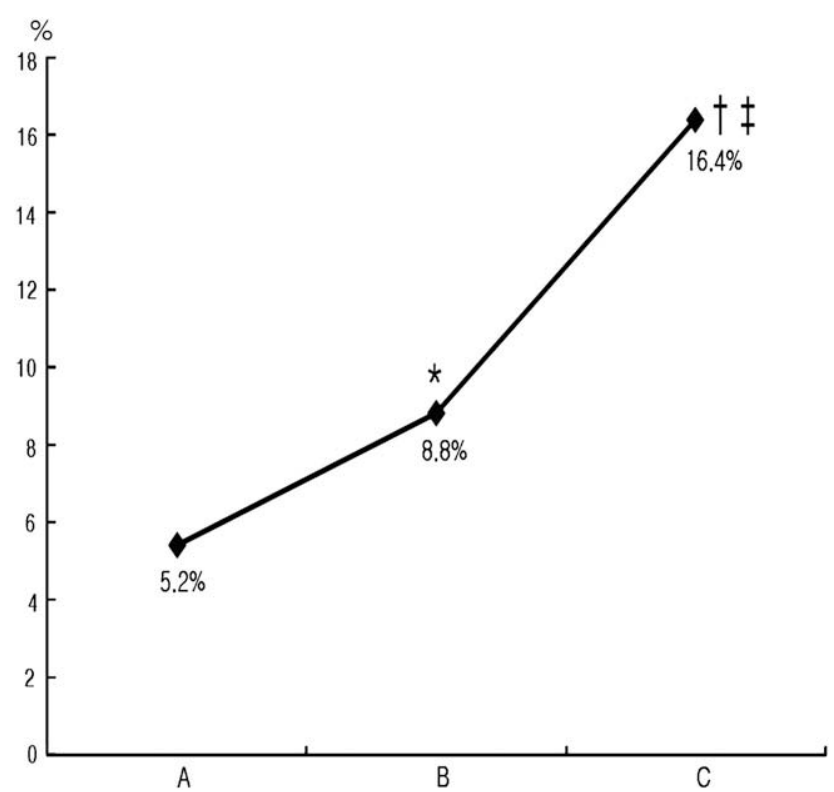

FIGURE 1. Significant tricuspid regurgitation development over time. A, Immediate postoperative evaluation; $\mathrm{B}$, evaluation at postoperative 1 year; $\mathrm{C}$, evaluation at the last follow-up. $* P>.1$ versus $\mathrm{A} ; \nmid P<.01$ versus $\mathrm{A}$; $\ddagger P<.01$ versus $\mathrm{B}$.

\section{Risk Factor Analysis of Late Significant TR}

The crude relative risks of FTR progression in terms of preoperative and operative variables are shown in Table 3. Univariate analysis showed FTR progression was associated with elderly patients, shorter ACC time, and omission of MAZE. The effect of preoperative atrial fibrillation, MV replacement (compared with repair), and no TV repair approached but did not quite reach significance in univariate analysis (Table 3). Multivariate analysis showed that older age, a rheumatic etiology of the MV disease, and no MAZE were independent predictors of FTR aggravation after successful MV surgery (Table 3).

\section{Effect of MAZE and TV repair in the AF group}

The protective effects of MAZE and TAP were further evaluated in the 168 patients with preoperative atrial fibrillation. Of those, 75 had MV surgery without TAP or MAZE, and the other 93 patients concomitantly had TAP $(n=20)$, MAZE $(\mathrm{n}=33)$, or TAP with MAZE $(\mathrm{n}=40)$. It was found that $36 \%(27 / 75), 20 \%(4 / 20), 3.0 \%(1 / 33)$, and $2.5 \%$ (1/ 40) of those patients developed significant TR during follow-up, respectively, and the difference of FTR progression following concomitant surgery is shown in Figure 3. Only 2 patients had significant TR following MAZE; an atrial fibrillation recurrence in 1 patient and a maintained sinus rhythm without right atrium (RA) mechanical activity in the other. Among the 73 patients who had MAZE, the normal sinus rhythm restoration rate was $87.7 \%(64 / 73)$ at $47.2 \pm 26.8$ months follow-up, 7 patients had recurring AF, and 2 patients maintained pacing rhythm. Most of the 64 patients who had restored normal sinus rhythm after MAZE were well maintained without antiarrhythmic medication; however, only $6.3 \%(4 / 64)$ and $3.1 \%(2 / 64)$ of patients required antiarrhythmic drugs a year after MAZE and the last followup, respectively.

\section{DISCUSSION}

Surgical treatment of mild FTR with concomitant MV disease remains a topic of debate. Although intraoperative inspection and palpation of the TV are frequently used to
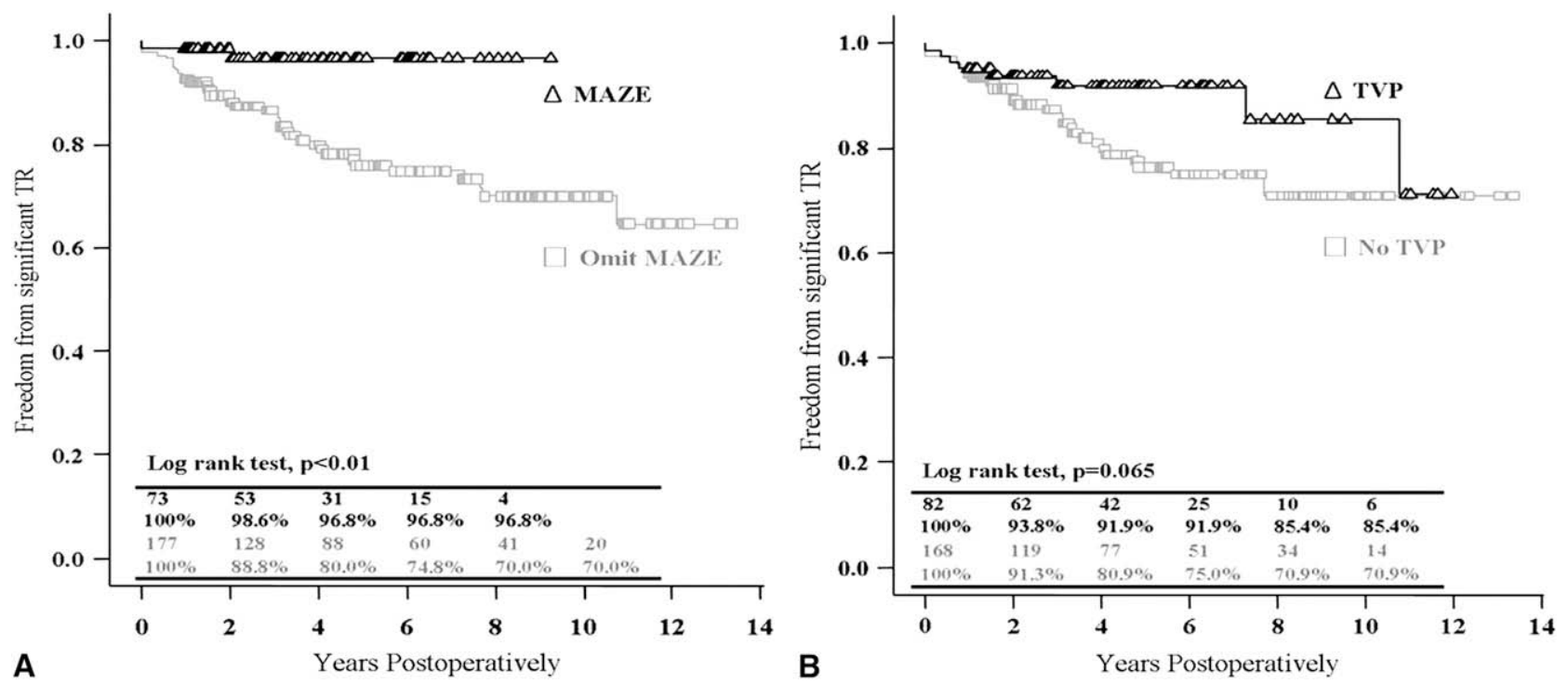

FIGURE 2. Kaplan-Meier analysis of long-term freedom from significant tricuspid regurgitation in (A) patients having Maze procedure (MAZE) versus patients not having Maze procedure (non-MAZE), and (B) patients having tricuspid annuloplasty $(T A P)$ versus patients not having tricuspid annuloplasty (non-TAP.) 
TABLE 3. Univariate and multivariate Cox regression analysis to identify factors associated with mild functional tricuspid valve regurgitation progression after mitral valve surgery

\begin{tabular}{lrrrrr}
\hline & CRR & $\boldsymbol{P}$ value & AHR & $\boldsymbol{P}$ value & $\mathbf{9 5 \%}$ CI \\
\hline Age (years) & 1.044 & $<.01$ & 1.05 & $<.01$ & $1.02-1.08$ \\
Atrial fibrillation & 1.812 & 0.13 & & & \\
Rheumatic etiology & 1.624 & .13 & 2.31 & .01 & $1.21-4.42$ \\
Preop EF (\%) & 1.002 & $>0.1$ & & & \\
Preop LA size (mm) & 1.010 & $>0.1$ & & & \\
Preop sPAP (mm Hg) & 1.004 & $>0.1$ & & & \\
MV pathology & 1.640 & .12 & & & \\
$\quad$ Mitral stenosis & & & & & \\
$\quad$ Mitral regurgitation & & & & & \\
CPB time (min) & 0.995 & .14 & & & \\
ACC time (min) & 0.989 & .02 & & & \\
MV procedure & 1.88 & .07 & & & \\
$\quad$ MV repair & & & & & \\
$\quad$ MV replacement & & & & & \\
No MAZE & 7.11 & $<.01$ & 7.90 & $<.01$ & $1.90-32.86$ \\
TV repair & 0.49 & 0.07 & & & \\
\hline
\end{tabular}

$n$, Number; $C R R$, crude relative risk; $A H R$, adjusted hazard ratio; $C I$, confidence interval; $E F$, ejection fraction; $L A$, left atrium; Preop, preoperative; $s P A P$, systolic pulmonary artery pressure; $M V$, mitral valve; $C P B$, cardiopulmonary bypass; $A C C$, aortic crossclamp; $T V$, tricuspid valve; $N S$, not significant.

evaluate the necessity of tricuspid repair, such methods can be subjective and may lead to incorrect management decisions. Recently suggested indicators for TV repair include the tricuspid annular diameter and the tricuspid annular function. ${ }^{8,12}$ However, preoperative assessment of the tricuspid annular diameter and function using echocardiography is unreliable and difficult as it can depend on the preload and afterload of the right ventricle at the time of measurement, and precise measurement of the tricuspid annulus is difficult and irreproducible due to the 3-dimensional structure of the TV.

Several reports suggest that TR can be regressive after successful mitral balloon valvuloplasty or surgery. It appears logical that significant FTR could be resolved if the transmitral pressure gradient and pulmonary artery pressure were markedly lowered. ${ }^{2}$ This concept is supported by findings that minimal right atrium (RA) enlargement and +1 to +2 regurgitation usually resolves after surgery on left-sided valve lesions, especially if the pulmonary hypertension resolves. ${ }^{13}$ In contrast, other studies reported that FTR could be progressive and should be treated even if preoperative TR is mild or grade II. ${ }^{6,14}$ In the present study, although only $5.2 \%$ of patients showed significant TR at the immediate postoperative echocardiography assessment, this number increased to $16.4 \%$ at the final follow-up. This finding suggests that TR can be progressive despite MV pathology being relieved.

Although some studies conclude ring annuloplasty is superior to suture annuloplasty for TR repair, ${ }^{15}$ others recommend ring annuloplasty for severe TR and suture annuloplasty for mild to moderate TR. ${ }^{14}$ In the present study, patients having ring annuloplasty and patients having suture

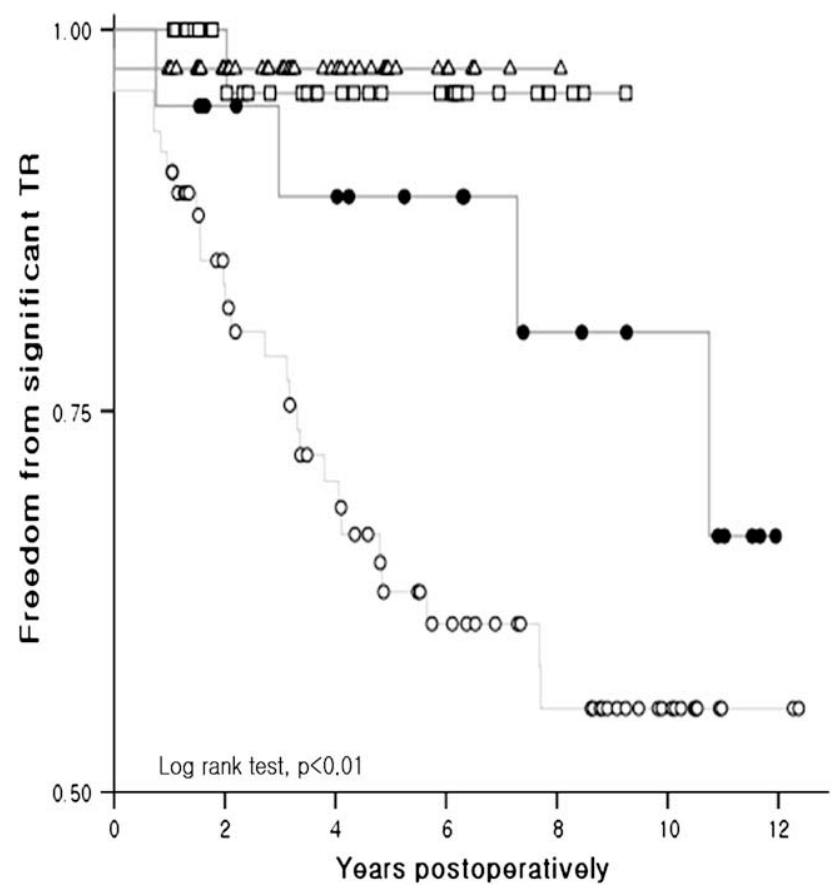

FIGURE 3. The effect of tricuspid annuloplasty and the Maze procedure on significant tricuspid regurgitation (TR)-free survival rates in patients with atrial fibrillation. $\bigcirc$, no tricuspid annuloplasty or Maze procedure; $\boldsymbol{~ , ~ t r i c u s p i d ~ a n n u l o p l a s t y ~ o n l y ; ~} \square$, Maze procedure only; $\Delta$, tricuspid annuloplasty and Maze procedure.

annuloplasty showed similar postoperative significant TR development rates. This may reflect that the present study population was exclusively patients with mild FTR and contained a small number of patients who had TR repair.

Previous studies identified several clinical and echocardiographic factors associated with development of late TR after left-sided valve surgery, such as atrial fibrillation, rheumatic etiology, older age, lower left ventricular ejection fraction, huge left atrium, and higher preoperative TR grade. ${ }^{6,16}$ Most risk factors for late FTR development are related to the chronic course of the left-sided valvular disease, and these chronic changes may contribute to irreversible changes to the tricuspid annulus and right ventricular function. The findings of the present study indicate that older age and a rheumatic etiology of the MV are strong predictors of significant TR progression after MV surgery even when the FTR is mild.

Because atrial fibrillation is a well-known risk factor for TR aggravation, ${ }^{6,9}$ a recent study suggested that sinus conversion resulting from MAZE can prevent FTR progression. ${ }^{9}$ In the present study, MAZE was found to be the most important predictor of FTR aggravation after MV surgery, and the incidence of significant TR progression by omission of MAZE increased 7.9 times. Although TV repair might alleviate mild FTR progression in patients with atrial fibrillation, MAZE had a more potent protective effect. 


\section{LIMITATIONS}

First, this study was subject to the limitations inherent to a nonrandomized, retrospective, observational data study of relatively small number of patients. Second, the present study contained several inherent selection biases because the decision to perform MAZE and/or tricuspid valvuloplasty was wholly dependent on surgeon preference. Third, ideally more precise data concerning MAZE should have been collected. Further studies are required to determine the relationship between FTR progression and restoration of sinus rhythm and/or effective atria contraction after MAZE in a larger study group. Fourth, precise assessments of the tricuspid annulus and right ventricular function were not conducted, thus sophisticated variables such as TV tethering distance and TV tethering area could not be estimated.

\section{CONCLUSIONS}

Mild FTR was shown to progress despite successful MV surgery. Older age and a rheumatic etiology of the MV disease were shown to be independent predictors of FTR progression. Although TV repair may protect against FTR progression, having MAZE was found to be a more important predictor of TR progression. From the present findings, we believe that MAZE should be performed not only for sinus restoration and alleviation of embolic complication after MV surgery but also for protecting against aggravation of mild FTR over the long term.

\section{References}

1. Braunwald NS, Ross J Jr, Morrow AG. Conservative management of tricuspid regurgitation in patients undergoing mitral valve replacement. Circulation. 1967;35:163-9.
2. Song JM, Kang DH, Song JK, Jeong YH, Lee CW, Hong MK, et al. Outcome of significant functional tricuspid regurgitation after percutaneous mitral valvuloplasty. Am Heart J. 2003;145:371-6.

3. Groves PH, Hall RJ. Late tricuspid regurgitation following mitral valve surgery. J Heart Valve Dis. 1992;1:80-6.

4. Kuwaki K, Morishita K, Tsukamoto M, Abe T. Tricuspid valve surgery for functional tricuspid valve regurgitation associated with left-sided valvular disease. Eur J Cardiothorac Surg. 2001;20:577-82.

5. Porter A, Shapira Y, Wurzel M, Sulkes J, Vaturi M, Adler Y, et al. Tricuspid regurgitation late after mitral valve replacement: clinical and echocardiographic evaluation. J Heart Valve Dis. 1999;8:57-62.

6. Matsuyama K, Matsumoto M, Sugita T, Nishizawa J, Tokuda Y, Matsuo T. Predictors of residual tricuspid regurgitation after mitral valve surgery. Ann Thorac Surg. 2003;75:1826-8.

7. Dreyfus GD, Corbi PJ, Chan KM, Bahrami T. Secondary tricuspid regurgitation or dilatation: which should be the criteria for surgical repair? Ann Thorac Surg. 2005;79:127-32.

8. Sugimoto T, Okada M, Ozaki N, Hatakeyama T, Kawahira T. Long-term evaluation of treatment for functional tricuspid regurgitation with regurgitant volume: characteristic differences based on primary cardiac lesion. J Thorac Cardiovasc Surg. 1999;117:463-71.

9. Kim HK, Kim YJ, Kim KI, Jo SH, Kim KB, Ahn H, et al. Impact of the Maze operation combined with left-sided valve surgery on the change in tricuspid regurgitation over time. Circulation. 2005;112:I14-9.

10. Currie PJ, Seward JB, Chan KL, Fyfe DA, Hagler DJ, Mair DD, et al. Continuous wave Doppler determination of right ventricular pressure: a simultaneous Doppler-catheterization study in 127 patients. J Am Coll Cardiol. 1985;6:750-6.

11. Lee JW, Choo SJ, Kim KI, Song JK, Kang DH, Song JM, et al. Atrial fibrillation surgery simplified with cryoablation to improve left atrial function. Ann Thorac Surg. 2001;72:1479-83.

12. Cain KC, Lange NT. Approximate case influence for the proportional hazards regression model with censored data. Biometrics. 1984;40:493-9.

13. Shemin RJ. Tricuspid valve disease. In: Cohn LH, Edmund LH, eds. Cardiac surgery in the adult. 2nd ed. New York: McGraw-Hill; 2003. p. 1003-4.

14. Duran CM. Tricuspid valve surgery revisited. J Card Surg. 1994;9:S242-7.

15. McCarthy PM, Bhudia SK, Rajeswaran J, Hoercher KJ, Lytle BW, Cosgrove DM, et al. Tricuspid valve repair: durability and risk factors for failure. $J$ Thorac Cardiovasc Surg. 2004;127:674-85.

16. Izumi C, Iga K, Konishi T. Progression of isolated tricuspid regurgitation late after mitral valve surgery for rheumatic mitral valve disease. J Heart Valve Dis. 2002; 11:353-6. 